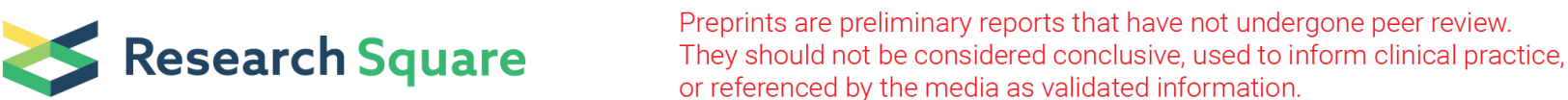

\section{LsaYAB7, A Homologous Gene of FILAMENTOUS FLOWER, Participating In The Regulation of Adaxial- Abaxial Polarity of Leaves In Lettuce}

Kangsheng Luo

China Three Gorges University

Dechun Zhang

China Three Gorges University

Zhaodong Zhai

Shandong Normal University

Xue Liu

Beijing Academy of Agriculture and Forestry Science (BAAFS)

Jun Zhou

Shandong Normal University

Bin Zhang

Beijing Academy of Agriculture and Forestry Science (BAAFS)

Dayong Li ( $\sim$ lidayong@nercv.org )

Beijing Academy of Agriculture and Forestry Science (BAAFS)

\section{Research Article}

Keywords: Lettuce, YABBY, Leaf, Adaxial-abaxial polarity, Expression profile

Posted Date: June 2nd, 2021

DOl: https://doi.org/10.21203/rs.3.rs-453206/v1

License: (c) (i) This work is licensed under a Creative Commons Attribution 4.0 International License.

Read Full License 


\section{Abstract}

Background: The plant-specific YABBY transcription factor plays important roles in the development of lateral organs, the establishment of adaxial-abaxial polarity and abiotic stress response. However, the function of $Y A B B Y$ gene family in lettuce (Lactuca sativa), an important leaf vegetable is still unclear yet.

Results: In this study, we analyzed the chromosomal distribution, gene structure and cis-transcriptional elements of the $Y A B B Y$ gene family in lettuce. Phylogenetic analysis of $Y A B B Y$ genes in lettuce, Arabidopsis, maize and rice classified them into five groups. In addition, the expression profile of $Y A B B Y$ genes in lettuce indicated that they may play distinct functions in different tissues and developmental processes. The subcellular localization analysis and transactivation assay showed that LsaYAB7 probably functions as a transcription factor in the nucleus. Furthermore, the ectopic expression of LsaYAB7 exhibited abaxially curled leaves, not only in Arabidopsis, but also in lettuce.

Conclusion: Altogether, $L s a Y A B 7$ is a key functional gene in determining the adaxial-abaxial polarity of lettuce leaves. Our study laid foundation for the molecular research of functional genes in lettuce.

\section{Background}

In the process of plant growth and development, diverse regulators participate in leaf morphology and development, such as GRF (GROWTH-REGULATING FACTOR), TCP (TEOSINTE BRANCHED1, CYCLOIDEA, and PROLIFERATING CELL NUCLEAR ANTIGEN BINDING FACTOR) and YABBY. Among them, YABBY genes encode plant-specific transcription factors that are especially widely distributed in seed plants [13]. They play an important role in the development of plant lateral organs, the establishment of adaxialabaxial polarity and abiotic stress response [4-6]. The YABBY transcriptional factor contains a C2-C2 zinc-finger domain in the $\mathrm{N}$-terminal and a high mobility group box in the $\mathrm{C}$-terminal region [7]. There are six members in YABBY gene family in Arabidopsis thaliana: YABBY1/FIL (FILAMENTOUS FLOWER), YABBY2, YABBY3, YABBY4/INO (INNER NO OUTER), YABBY5 and CRC (CRABS CLAW) [8]. These six $A t Y A B$ genes have distinct and redundant functions in regulating morphogenesis of plant organs [9]. Accordingly, CRC and INO are usually called "flower-specific YABBY genes", while FIL, YABBY2, YABBY3 and $Y A B B Y 5$ are called "nutritional $Y A B B Y$ genes" [10]. FIL, YABBY2 and YABBY3 are expressed in polar manner and functionally redundant in all lateral organs including cotyledons, petals, sepals, carpels and stamens with different expression levels [9]. FIL, YABBY2 and YABBY3 express respectively at a high, moderate and low level and jointly determines the fate of abaxial cells [9-12]. $C R C$ is specifically expressed in the carpels and nectaries, and $c r c$ mutant shows non-fusion of carpels and abnormal developed nectaries [7]. While INO is expressed in the abaxial region of outer integument, and INO mutation leads to loss of polarity differentiation in ectoovule [13]. The FIL/YAB3 double mutant shows linear cotyledons and leaves, and abnormal vascular tissue development [2]. The triple mutant fil/yab3/yab5 and quadruple mutant fil/yab2/yab3/yab5 are short and dense, lack apical dominance and polarity of lateral organs $[2,9]$. $Y A B 2$ shows functional redundancy with $Y A B 3$ and $C R C$, which regulate leaf development and participating in the synthesis of cell wall [14]. 
Leaf development, and in particular, the establishment of the adaxial-abaxial polarity, is regulated by a precise genetic network. phantastica (phan) in Antirrhinum is the first mutant affecting the leaf adaxialabaxial polarity, with the leaves showing more and more radial symmetry, and the leaf at the top of the stem became needle-like $[15,16]$. In Arabidopsis thaliana, the fate of the abaxial cells is comprehensively determined by the interaction between YABBY, KANADI and auxin response factor (ARF3/4) and miR165/166 [9, 11, 17-21]. Upon establishment of the adaxial-abaxial polarity $Y A B B Y$ is initially expressed in the leaf primordium, and then fixed in the abaxial region, acting downstream of KANADI, which functions antagonistically to the adaxially expressed $H D$-ZIPIII gene that also limited by miR165/166 [18, 22-27]. AS1 and AS2 promoted the expression of HD-ZIPIII in the adaxial side and inhibited the expression of miR165/166, Kanadi and $Y A B B Y$ genes in the adaxial side[28, 29]. TAS3 transacting short interference RNA (TAS3 ta-siRNAs) limits the expression of ARF3/4 genes to the abaxial side [30-32].

At present, many $Y A B B Y$ genes in different plants have been found to play an important role in leaf adaxial-abaxial polarity establishment, floral organ recognition, and SAM development. In rice, OSYAB1 participates in the feedback regulation of GA synthesis by the specifical binding to the cis-acting element of GA3ox2 promoter [33]. OsYAB2 controls shedding [34]. OsYAB3 was closely related to maize ZmYAB14 and Arabidopsis FIL. OsYAB3-RNAi showed twisted leaves without ligule and auricle [35]. OsYAB4 overexpression in Arabidopsis exhibited retardation of development, loss of apical dominance at flowering stage, more stems, tufted flowers and less fruiting, similar to FIL/YAB3 overexpression lines [36]. DROOPING LEAF $1(D L 1)$ plays a regulatory role in midvein formation and carpel development in rice [37]. The $C R C$ homologous gene in maize, regulates plant structure by affecting leaf length and width, leaf angle, internode length and diameter [38]. In tomato, a YABBY-like FAS protein influences the formation of carpels [39]. In addition, the $Y A B B Y$ genes are also associated with abiotic tolerance such as drought, salt and ABA stress in soybean [6]. The negative regulative role of $Y A B B Y$ gene under drought and salt stress is also reported in upland cotton [40].

Altogether, $Y A B B Y$ genes are pivotal regulators in leaf development [41], however, in lettuce (Lactuca sativa L.), a popular leafy vegetable in the world, and corresponding studies on the $Y A B B Y$ gene function are rarely reported, due to the relatively slow progress in development biological research in lettuce. In this study, we carried out the studies on the $Y A B B Y$ genes in lettuce. Nine $Y A B B Y$ genes were identified in lettuce, and furthermore, their gene structure, protein conservative motif, cis-acting elements and expression profile in different tissues were studied. Moreover, the regulatory roles of some $Y A B B Y$ gene in leaf development, especially in adaxial-abaxial polarity establishment in lettuce were revealed by genetic study. This study laid the foundation for further exploration on the molecular regulatory network of lettuce leaf development.

\section{Results}

\section{Identification of the YABBY gene family in lettuce}


In order to identify YABBY gene family in lettuce, both BLASTP and Hidden Markov Model (HMM) searches were performed. Initially, the amino acid sequences of six YABBY transcription factors in Arabidopsis were used as query sequences for performing a BLASTP search in lettuce genome database. Additionally, HMM profiles of the YABBY (PF04690) domains were searched against the lettuce genome database (https://phytozome.jgi.doe.gov). Finally, a total of 9 candidate YABBY genes were identified in lettuce. Further lettuce database searching using these candidate YABBYs as queries against the draft genome and predicted mRNAs resulted in no further hits. Thus, in total, 9 proteins were identified as LsaYABs, and their coding genes were designated as $\angle S a Y A B 1$ to $L s a Y A B 9$, according to their distribution in the lettuce genome (Table 1). Based on the location information of 9 LsaYABs genes on chromosomes, it was found that 9 LsaYABs genes were distributed on 6 chromosomes (chromosomes 3, 4, 5, 6, 7 and 8) (Fig. 1A). Among them, Chromosomes 3 caries three $L$ saYABs, chromosome 7 has two, whereas chromosomes 4, 5, 6, and 8 each contains one $L s a Y A B$ gene. By comparing the protein sequences of $\angle s a Y A B s$, it was found that all of them contained conserved YABBY and C2-C2 zinc-finger domains (Fig. 1B).

The proteins encoded by the $\angle s a Y A B s$ were predicted to show significant differences in their sizes and physicochemical properties (Table 1). Their predicted protein lengths varied from 168 to 228 amino acids and molecular weights ranged from $18.61 \mathrm{kDa}$ to $25.09 \mathrm{kDa}$ (Table 1). Exon numbers of the $\angle s a Y A B s$ genes ranged from 3 to 7, with $L s a Y A B 2$, LsaYAB3, LsaYAB4, LsaYAB7 containing seven exons and $\angle s a Y A B 5$ containing only three. Their lengths of nucleotides are relatively short, none of them was longer than $700 \mathrm{bp}$. In addition, YABBY protein sequences showed large variations in predicted isoelectric point (pl) values (ranging from 6.29 to 9.43 ).

Table 1

Characteristics of the YABBY proteins in lettuce

\begin{tabular}{|llllllll|}
\hline Name & Accession No. & Chr & $\begin{array}{l}\text { CDS } \\
\text { (bp) }\end{array}$ & Exon No. & $\begin{array}{l}\text { Length } \\
\text { (aa) }\end{array}$ & $\begin{array}{l}\text { MW } \\
\text { (KDa) }\end{array}$ & PI \\
\hline LsaYAB1 & Lsat_1_v5_gn_0_27800.2 & Chr04 & 507 & 5 & 168 & 18.61 & 6.29 \\
\hline LsaYAB2 & Lsat_1_v5_gn_3_3040.1 & Chr03 & 687 & 7 & 228 & 25.09 & 8.15 \\
\hline LsaYAB3 & Lsat_1_v5_gn_3_53601.1 & Chr03 & 552 & 7 & 183 & 19.97 & 9.28 \\
\hline LsaYAB4 & Lsat_1_v5_gn_3_128981.1 & Chr03 & 666 & 7 & 221 & 24.67 & 7.27 \\
\hline LsaYAB5 & Lsat_1_v5_gn_5_94941.2 & Chr05 & 561 & 3 & 186 & 20.62 & 9.28 \\
\hline LsaYAB6 & Lsat_1_v5_gn_6_2400.1 & Chr06 & 669 & 6 & 222 & 24.57 & 9.43 \\
\hline LsaYAB7 & Lsat_1_v5_gn_7_9041.1 & Chr07 & 672 & 7 & 223 & 24.60 & 7.21 \\
\hline LsaYAB8 & Lsat_1_v5_gn_7_43360.1 & Chr07 & 600 & 6 & 199 & 22.02 & 6.53 \\
\hline LsaYAB9 & Lsat_1_v5_gn_8_58340.3 & Chr09 & 648 & 5 & 215 & 24.02 & 8.66 \\
\hline
\end{tabular}


In order to study the phylogenetic relationship of the nine $\angle s a Y A B s$ genes, a Neighbor-Joining phylogenetic tree containing $Y A B B Y$ genes from Arabidopsis, lettuce, rice and maize was constructed by using MAGE 7.0 software (Fig. 2).In this analysis, 9 LsaYABs genes were divided into 5 groups, named FIL/YAB3, YAB2, CRC, INO and YAB5, respectively (Fig. 2) [33, 42]. Among them, FIL/YAB3 contains the largest number of family members, with four $L s a Y A B s$, namely $L s a Y A B 2, L s a Y A B 4$, LsaYAB6 and $\angle s a Y A B 7$ belonging to this group (Fig. 2). YAB5 contains three members from lettuce, namely $L s a Y A B 5$, $\angle s a Y A B 8$ and $\angle s a Y A B 9 ; C R C$ and INO each contain only one member from lettuce (Fig. 2). However, there is no $L s a Y A B s$ in YAB2 group, indicating that functional loss may have occurred in genome replication events. Monocots (rice and maize) and dicotyledous (Arabidopsis and lettuce) have low gene similarity and were separated into each group. Most of the $\angle s a Y A B$ genes are closer to those in Arabidopsis, except for $L s a Y A B 1$ and $\angle s a Y A B 3$ (Fig. 2). It implied that these two YABBY genes might be involved in the conserved function of monocots and dicotyledous.

Gene structures often diverges during the evolution of multi-gene families. Therefore, we analyzed the gene structures and conserved motif characteristics of the LsaYABs. The phylogenic tree of LsaYABs was used in gene structures and conserved motif analysis (Fig. S1). As shown in Fig. S2A, the LsaYABs within each group had similar exon-intron organizations. Most $L s a Y A B$ genes harbored 5-7 exons, with the exception of $\angle s a Y A B 5$, which has 3 exons. Tools of the MEME web server (http://meme.sdsc.edu/meme/meme.html) were used to analyze motif distributions and to conduct domain predictions. A total of 8 motifs were identified in the LsaYABs (Fig. S2B). The predicted motif models are listed in Fig. S3. LsaYABs contain at least two domains, with Motif 2 representing the zinc finger domain and Motif 1 the YABBY domain. The YABBY gene family has a very close relationship in evolution and distribution. LsaYABs in the same group usually show similar protein conservative motif types and sequences within. For example, $L s a Y A B 4$ and $L s a Y A B 7$ have 7 identical motifs, with same sequence. $\angle s a Y A B 2$ and $\angle s a Y A B 6$ have six identical motifs in exactly the same order. Among them, Motif 5 and Motif 8 exist in $L s a Y A B 4$ and $L s a Y A B 7$, Motif 6 exists in $L s a Y A B 5$ and $L s a Y A B 8$. These motifs, which exist specifically in different subfamilies, may play important roles in the functional differentiation of YABBY gene family proteins.

The cis-elements in gene promoter play critical roles in the initiation of gene expression. Investigation of cis-regulatory elements in LsaYAB genes was performed within $2000 \mathrm{bp}$ upstream from the putative translation start site using the PlantCARE database. Various cis-elements related to hormone and stress responses were identified in the $L S a Y A B$ promoters (Fig. S4). $L$ saYABs contain a large number of cisacting elements related to light responsiveness (G-Box, MRE, GT1-motif, ACE, 3-AF1 binding site) and MeJA-responsiveness (CGTCA-motif, TGACG-motif). Additionally, it contains many cis-acting elements related to stress resistance (drought and low temperature response) and hormone response (abscisic acid and gibberellin induction). $\angle s a Y A B 3, \angle s a Y A B 6$, and $\angle s a Y A B 7$ contain meristem expression element (CATbox), indicating that these genes may play roles in the development of plant meristems. LsaYAB3 also contains cis-regulatory element related to flavonoid biosynthetic genes regulation element (Fig. S4). The $\angle s a Y A B$ genes in the same phylogenetic cluster showed moderate consistency in their distributions of the cis-elements, indicating that the promoters of these $\angle$ saYABs diverged. 
Phylogenetic tree was constructed for 9 Lactuca sativa, 6 Arabidopsis thaliana, 13 Maize and 8 Oryza sativa YABBY proteins. There were 5 phylogenetic clusters, highlighted with different colors, designated as CRC, INO, YAB2, YAB5, FIL/YAB3. Numbers at the nodes indicate bootstrap values; values lower than $30 \%$ are not shown.

\section{Expression profiles of YABBY genes in different tissues}

To characterize the function of the $\angle s a Y A B$ genes, we firstly evaluated their expression levels in the root, stem, cotyledon, leaf, bud, flower and seed of lettuce. It was revealed that most of the genes showed relatively high expression in the flower and seed, while low expression in the in root and stem (Fig. 3). LsaYAB1, the putative homologous gene of Arabidopsis INO, specifically expressed in the flower. And LsaYAB3, the putative homologous gene of Arabidopsis CRC, expressed highly in bud and flower. LsaYAB7 is a putative homologous gene of Arabidopsis FIL and it has the highest expression in floral organs, which is consistent with the expression pattern of $A t F I L$ gene [9]. LsaYAB7is also expressed in leaves and floral organs.

To investigate the expression pattern of $\angle s a Y A B 7$, we constructed a vector containing $\beta$-glucuronidase (GUS) reporter gene driven by the promoter of $L s a Y A B 7$ (2 kb sequence upstream of e the initiation codon of $L s a Y A B 7)$, and genetically transformed into Arabidopsis. GUS activity was observed in the cotyledons and the root tip at cotyledon-stage (Fig. 3B). After two true leaves were full expanded, GUS signal was seen in petioles of cotyledons, but not in cotyledons and true leaves (Fig. 3B). The GUS activity in the initiation of lateral roots were also observed at two-leaf-stage (Fig. 3B). GUS staining was also found in the apical stem and the petioles, but not the cotyledons at the four-leaf-stage (Fig. 3B). These results indicated that $L s a Y A B 7$ expressed in the tissues undergoing cell proliferation, which was consistent with that of AtFIL.

\section{LsaYAB7 putatively functions as a transcription factor in the nucleus}

LsaYAB7 in FIL group shows a similar expression pattern to AtFIL, an important regulator of leaf development. Therefore, we further investigate the function of $L s a Y A B 7$. As the LsaYAB proteins are putative TFs, subcellular location observation and transactivation assay of LsaYAB7 were firstly investigated. We constructed the 35S::LsaYAB7-GFP vector and transformed it into lettuce protoplast cells. The empty vector was used for control. As shown in Fig. 4A, green fluorescence of GFP was observed within the nucleus in the protoplast cell transformed by 35S.:LsaYAB7-GFP. While the protoplast cell transformed by empty vector exhibited ubiquitous green fluorescence. Agrobacterium with recombinant plasmid 35S.:LsaYAB7-GFP was transfected into tobacco leaves too. Also, the fluorescence signal was detected in the nucleus (Fig. 4B), indicating that the LsaYAB7-GFP localized to the nuclear. In the transcriptional activity, full-length $L$ saYAB7 was constructed into yeast expression vector, pGBK-T7. The empty vector pGBK-T7 and pGBK-T7-OsMYB103L which was proved to have transcriptional activity were designed as the negative and positive control, respectively [47]. These recombinant plasmids were transformed into yeast strain Y2HGold. They showed similar growth states without Tryptophan (Trp) under different diluted concentration (Fig. 4C), indicating the transformation made few influences on 
yeast growth. The yeast cells expressed full-length $\angle s a Y A B 7$ could grow with $A b A$ (Aureobasidin A) and turn blue with X-a-galactoside, similar to the positive control, indicating that $\angle s a Y A B 7$ had transcriptional activity in yeast (Fig. 4 C). These results implied that $\angle s a Y A B 7$ probably functioned as a transcriptional factor in the nucleus.

\section{LsaYAB7 functioned in leaf adaxial-abaxial polarity establishment in Arabidopsis and lettuce}

To further investigate the function of the $\angle s a Y A B 7$ gene, we constructed the over-expression plasmid of $\angle s a Y A B 7$ gene driven by $35 \mathrm{~S}$ promoter, and respectively transformed into Arabidopsis using Agrobacterium-mediated transformation method and lettuce using leaf disc method. A total of 12 transgenic lines were obtained and three of which with relatively higher expression (Fig. 5A) were further used for phenotype investigation. Compared with transgenic negative line (CK) plants, all homozygous transgenic lines (10-day-old) from T1 generation exhibited outward curled cotyledons (Fig. 5B); along with the growing process, the leaves curled abaxially became long-narrow, and this phenotype turned more obvious with the increase of the leaf ages. In addition, the LsaYAB7-OE plants were short, and the flowering stage was delayed (Fig. 5). Conclusively, we demonstrated that over-expression of LsaYAB7 in Arabidopsis caused abaxialization of the leave, which is consistent with the phenotype of AtFIL overexpression [43].

In the lettuce transformation, PCR identification showed that the $L s a Y A B 7$ gene has been successfully transformed into the lettuce genome (Fig. 6). qRT-PCR detection of the relative expression of the LsaYAB7 gene in the transgenic plants showed that the expression of $L s a Y A B 7$ was significantly up-regulated compared with that in the transgenic negative line (CK) (Fig. 6A). Compared with non-transgenic plants, the leaves of transgenic lines curled abaxially (Fig. 6B). These results indicated that the overexpression of $\angle s a Y A B 7$ affected the leaf adaxial-abaxial polarity and led to outward curling of leaves in lettuce, which was consistent with the phenotype of ectopic expression of $L s a Y A B 7$ in Arabidopsis.

\section{Discussion}

The YABBY gene family plays an important role in abaxial-adaxial polarity establishment, carpel morphogenesis, shoot apical meristem restriction, floral differentiation, and development of lateral organs [22]. In this study, we firstly identified 9 YABBY genes in lettuce and cloned $L s a Y A B 7$, the homologous gene of AtFIL, which regulates leaf abaxial-adaxial polarity in Arabidopsis and lettuce. Overexpressed $L s a Y A B 7$ in Arabidopsis thaliana and lettuce could result in obvious curled leaves, which is consistent with the phenotype of AtFIL overexpression line in Arabidopsis. According to the phylogenetic analysis, the YABBY genes in lettuce, Arabidopsis thaliana, rice and maize could be divided into five groups (Fig. 2), among which the group of FIL/YAB3 contains the most of LsaYABs, as well in rice and maize $[33,42]$. There was no $L s a Y A B$ genes found in $Y A B 2$ group, which might be lost during the interspecific differentiation. In addition, it was also found that the gene structures and conserved motifs of $\angle s a Y A B s$ in the same group were conserved to a certain extent (Fig. S2). 
The functions of $A t Y A B$ genes were found to be involved in the development of abaxial tissue of lateral organs. In this study, the expression profiles of $9 \angle s a Y A B$ genes in lettuce were analyzed and $\angle s a Y A B$ genes were identified to be widely expressed in various organs (Fig. 3). It showed that the expression of $\angle s a Y A B$ gene was significantly up-regulated in lateral organs such as leaves, flowers and seeds, which implied that $\angle s a Y A B$ gene, similar to $A t Y A B$ genes, plays a certain role in the development of lateral organs. Nine $\angle s a Y A B s$ were all found to be highly expressed in flowers, of which $L s a Y A B 1$ was detected to be expressed specifically in flowers (Fig. $3 A$ ). $L s a Y A B 1$ is a homologous gene of $A t / N O$, which has the highest expression in abaxial region of the outer integument [12]. It suggested that $\angle s a Y A B 1$ might have the same functions with its homologous gene AtINO. LsaYAB7 was mildly expressed in cotyledon and true leaf. However, its expression reached the highest level in the bud and gradually decreased at flowering stage (Fig. 3A). It might be similar with the expression pattern of AtFIL in Arabidopsis, that its expression was accumulated in the flower primordium at the beginning and gradually restricted to the abaxial region of the flower organs with the differentiation of floral organs [9].

The amino acid sequence alignment of LsaYABs and AtYABs showed that all of the YABBY genes contained two highly conserved protein domains, C2-C2 zinc finger and YABBY, which might be associated with similar molecular functions (Fig. 1B). However, the expression patterns of LsaYABs showed diversity in different organs and development stages, which suggested that they may function in different temporal and spatial ways (Fig. 3). The transcription elements analysis of $\angle$ saYABs showed that the putative promoters of $\angle s a Y A B s$ contains a large number of light response-related cis-acting elements, indicating that the expression of $\angle s a Y A B s$ may be greatly influenced by light. In addition, some $\angle s a Y A B s$ have gibberellin, abscisic acid, methyl jasmonate, ethylene, low temperature and drought response elements in their putative promoter sequences. Abscisic acid, methyl jasmonate and salicylic acid play important roles in response to abiotic stress and disease resistance. It may indicate that some $L s a Y A B S$ were not only related to the development of plant lateral organs, but also closely related to hormone signaling transduction and stress responses. Cis-acting elements related to regulate flavonoid synthesis were also found in the putative promoter of $\angle s a Y A B 3$, indicating that $\angle s a Y A B 3$ might also be involved in the regulatory pathway of flavonoid synthesis.

\section{Conclusions}

Although there have been lots of researches on the functions of YABBY genes, the YABBY genes in lettuce have not been identified and characterized yet. In this study, the members of YABBY gene family in lettuce were firstly identified and conserved protein domains, gene structures, conserved motif and cis-acting elements of these $L s a Y A B s$ were also analyzed. The overexpressed $L s a Y A B 7$, a homologous gene of AtFIL, both in Arabidopsis and lettuce resulted in the curled leaves and tufted flowers like the overexpression lines of AtFIL in Arabidopsis. These results in this research have laid a foundation for the functional investigation of the YABBY genes in lettuce. However, the phenotypes of $L s a Y A B 7$ loss-offunction lines in lettuce, the downstream regulation genes of $\angle s a Y A B 7$ and the functional analysis of other $\angle s a Y A B$ genes, would need a further study. This study is of great significance to analyze the 
molecular mechanism of leaf shape variation in lettuce and laid a foundation for molecular breeding of different leaf shapes in lettuce.

\section{Methods}

\section{Plant materials}

'L3', a loose-leaf type cultivar of lettuce (Lactuca sativa L.), was used in this study. The seeds of 'L3' were provided by Beijing vegetable Technology Research Center. Spread lettuce seeds evenly in petri dishes covered with filter paper and add $\mathrm{ddH}_{2} \mathrm{O}$ to keep the filter paper moist. After 1 day of treatment at $4{ }^{\circ} \mathrm{C}$, the lettuce seeds were transferred to a light incubator, and the seeds could sprout after 2 days. The germinated seeds were transferred to a burrow tray to raise seedlings. When 5-6 true leaves of lettuce were grown, they were transplanted in a protected field (seedling spacing 6-8 cm) and watered every 5-7 days.

\section{Genetic transformation}

The lettuce transformation method were modified according to the previous description [44]. To obtain the lettuce explants used for genetic transformation, we firstly soaked the full lettuce seeds in $70 \%$ ethanol for 1 minute and washed them with aseptic water for 3 times, then added $10 \%$ bleaching disinfection water for 15 minutes and washed them with aseptic water for 5 times. Finally, the sterile seeds were sown on Murashige and Skoog (MS) solid medium and cultured at $25{ }^{\circ} \mathrm{C}$ for 16 hours to grow two cotyledons. The cotyledons of lettuce seedlings were taken using scissors and were co-cultured with Agrobacterium tumefaciens for $15 \mathrm{~min}$. The infected leaves were placed on co-culture medium MS1 (MS with $0.5 \mathrm{mg} / \mathrm{L}$ 6-BA and $0.1 \mathrm{mg} / \mathrm{L} \mathrm{NAA}$ ) at $25^{\circ} \mathrm{C}$ dark condition for $48 \mathrm{~h}$ for 2 days. Then the leaves were transferred into differentiation medium MS2 (MS with $0.5 \mathrm{mg} / \mathrm{L} 6-\mathrm{BA}, 0.1 \mathrm{mg} / \mathrm{L} \mathrm{NAA}, 50 \mathrm{mg} / \mathrm{L}$ Kan and 50 $\mathrm{mg} / \mathrm{L} \mathrm{Tim}$ ) at $25^{\circ} \mathrm{C}, 16$-h-light/8-h-dark condition for about 2-4 weeks to obtain kana-resistant differentiation seedlings. The buds were cut off, transferred to MS3 medium (MS with $0.046 \mathrm{mg} / \mathrm{L}$ 6-BA, $0.026 \mathrm{mg} / \mathrm{L} \mathrm{NAA}, 50 \mathrm{mg} / \mathrm{L} \mathrm{Kan}$ and $50 \mathrm{mg} / \mathrm{L} \mathrm{Tim}$ ) for culture, and then transferred to the medium MS4 (MS with $20 \mathrm{mg} / \mathrm{L} \mathrm{Kan}$ and $50 \mathrm{mg} / \mathrm{L} \mathrm{Tim}$ ) for roots generation. After the root system is developed, the seedlings were transferred to soil.

\section{Identification of $Y A B B Y$ gene family in lettuce}

In order to identify the $Y A B B Y$ genes in the genome of lettuce, 15 full-length amino acid sequences of AtYABBY members were obtained from Arabidopsis thaliana genome database TAIR (https://www.arabidopsis.org). Using them for inquiry sequences to blast the protein sequence of all members of YABBY gene family in lettuce was obtained by BLASTp comparison search in Phytozome v12 (http://phytozome.jgi.doe.gov/pz/). Then, we used SMART (http://smart.embl-heidelberg.de) and PFAM (http://pfam.xfam.org) to analyze the sequence of the candidate members, excluding the members that do not contain conservative domains, all members of the YABBY transcription factor family of lettuce were finally obtained. The physical and chemical properties of lettuce YABBY proteins, such as 
molecular weight and isoelectric point, were analyzed on ExPASy website (https://web.expasy.org/protparam/). Multiple sequence alignment analysis of 9 LsaYABs protein sequences was carried out by DNAMAN software.

\section{Prediction of promoter cis-elements in LsaYABs}

The 2000 bp regions upstream of the start codon of $L s a Y A B s$ were downloaded from Phytozome V12. The cis-acting elements of these sequences were predicted on website PlantCare (http://bioinformatics.psb.ugent.be/webtools/PlantCare/html/), and were predicted.

\section{Construction of phylogenetic tree}

Multiple sequences alignment of YABBY proteins in lettuce were carried out using DNAMAN software, and the phylogenetic tree was constructed by Neighbor-Joining method using MEGA 7.0 software. The Bootstrap value was set to 1000 times, and P-distance and Complete Deletion models were selected for analysis.

\section{RNA extraction and qRT-PCR}

A certain amount of plant tissue was taken with RNase-free 's $2 \mathrm{~mL}$ centrifuge tube and quickly placed in a liquid nitrogen tank. Total plant RNA was extracted by polysaccharide polyphenol plant total RNA extraction kit (TIANGEN). RNA was reverse transcribed into cDNA using a reverse transcription kit (TransScript ${ }^{\circledR}$ One-Step gDNA Removal and cDNA Synthesis SuperMix). The synthesized first strand CDNA was diluted 5 times for real-time quantitative PCR amplification. The reaction was carried out by Roche480 real-time quantitative PCR system. The YABBY genes and reference Actin gene were amplified in each sample and three biological repeats were set in each sample. The preparation and reaction conditions of the system refer to the kit specification TransStart ${ }^{\circledR}$ TopGreen qPCR Super Mix Kit (TransGen, Beijing, China). The fluorescence signal of the first 15 cycles of the PCR reaction was used as the fluorescence background signal. The $\mathrm{Ct}$ value of the sample was used to calculate the relative expression of the gene by $2^{-} \Delta \Delta C T$. Sequences of primers used for qRT-PCR are listed in Supplementary Table S1.

\section{Subcellular localization and transactivation assay of $L s a Y A B 7$}

According to the CDS sequence of $\angle s a Y A B 7$ downloaded from Phytozomev12 database, specific primers

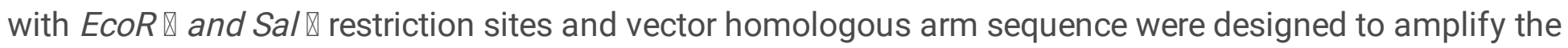
cDNA fragment of target gene (excluding stop codon). After amplified fragment was ligated into the pSAT6-EGFP-N1 vector digested by ECOR $\$ and Sal $\otimes$ by the method of homologous recombination. The Agrobacterium containing recombinant plasmid LC-LsaYAB7-GFP and empty vector were injected into tobacco leaves. After $12 \mathrm{~h}$ and $48 \mathrm{~h}$, the tobacco leaves were observed under a Nikon A1R/A1 confocal microscope with wavelengths of $514 \mathrm{~nm}$ (Nikon, Tokyo, Japan). 
In order to detect the transcriptional activation activity in yeast cells, its full-length coding sequence, $\mathrm{N}$ terminal DNA binding domain and C-terminal activation domain of LsaYABBY7 were cloned into pGBKT7 vector at $E c o \mathrm{R} \mathrm{I}$ and $B a m H \mathrm{I}$ sites by homologous recombination. The empty vectors pGBKT7 were used as negative control. All of these vectors were transformed into yeast Y2HGold containing AUR1C and MEL1 reporter genes respectively. After transformation, the yeast clones were cultured on SD/-Trp and $\mathrm{SD} /$-Trp/ABA/X-a-GAL plates at $30{ }^{\circ} \mathrm{C}$ for 2-4 days. The primers used for vector construction were listed in Table $\mathrm{S} 1$.

\section{Plasmid construction and plant transformation}

The cloning primers of $L s a Y A B 7$ was designed based on the genome sequences of lettuce (Lactuca sativa V8) from the Phytozome (https://phytozome.jgi.doe.gov). The coding sequences (CDS) of $\angle$ saYAB7 was cloned and constructed into the overexpression vector $\mathrm{pEZR}(\mathrm{K})$-LC driven by $35 \mathrm{~S}$ promoter using $2 X$ Seamless Cloning Mix (Biomed, CL117-01, Beijing). The primers used for vector construction were listed in Table S1.

The $L$ saYAB7 CDS were PCR-amplified and introduced into the LC vector. The recombinant constructs were transformed into Arabidopsis and Lettuce using Agrobacterium-mediated transformation following the floral dip method and the leaf disk method respectively $[44,45]$. Transgenic plants were then screened on solid MS medium containing $50 \mu \mathrm{g} / \mathrm{ml}$ kanamycin (Kana). Resistant seedlings were transferred to soil and further verified by PCR and qRT-PCR.

\section{Histochemical GUS staining}

The $L s a Y A B 7$ promoter containing 2000 bp upstream from ATG were cloned into modified pCambia vector to drive GUS gene expression. The recombinant plasmid was transformed into Arabidopsis and the tissues from different developmental stages of transgenic Arabidopsis plants were used for GUS staining. The tissues were stained in the solution (50 mM phosphate buffer, $\mathrm{pH} 7.0$, containing $1 \mathrm{mM}$ Glc and $10 \%$ methanol) at $37^{\circ} \mathrm{C}$ for $16 \mathrm{~h}$. Then the tissues were immersed in $70 \%$ ethanol for several hours to remove chloroplast. Finally, the tissues were subjected to microscopic observation using a light microscope (SMZ1500; Nikon), and digital images were taken (Fi1; Nikon).

\section{Abbreviations}

MW: Molecular weight

ARF: auxin response factor

DL1: DROOPING LEAF 1

TCP: TEOSINTE BRANCHED1, CYCLOIDEA, and PROLIFERATING CELL NUCLEAR ANTIGEN BINDING FACTOR 
FIL: FILAMENTOUS FLOWER

INO: INNER NO OUTER

CRC: CRABS CLAW

TF: transcription factor

MS: Murashige and Skoog

pl: Isoelectric point

qRT-PCR: Quantitative real-time polymerase chain reaction

GRF: GROWTH-REGULATING FACTOR

AbA: Aureobasidin A

\section{Declarations}

\section{Ethics approval and consent to participate}

Not applicable.

\section{Consent for publication}

Not applicable.

\section{Availability of data and material}

The links to the databases used in this study were listed as follows.

1. Arabidopsis thaliana genome database TAIR (https://www.arabidopsis.org)

2. Phytozome v12 (http://phytozome.jgi.doe.gov/pz/)

3. SMART (http://smart.embl-heidelberg.de)

4. PFAM (http://pfam.xfam.org)

5. ExPASy website (https://web.expasy.org/protparam/)

6. PlantCare (http://bioinformatics.psb.ugent.be/webtools/PlantCare/html/)

The public access to all these databases is open.

\section{Competing interests}

The authors declare that they have no competing interests 


\section{Funding}

This work was supported by grants from the Innovation and Development Program of Beijing Vegetable Research Center [KYCX202002-01 and KYCX201901-01], the Beijing Academy of Agriculture and Forestry Science [KJCX20200113]. The funding was used for performing experiments and paying salaries of researchers.

\section{Authors' contributions}

D. L, D. Z., J. Z., and B. Z. designed experiments. K. L., Z. Z. and B. Z. identified and characterized the YABBY genes family in lettuce. K. L., Z. Z., and X. L. carried out the experiment. K.L., B.Z., and D. L. wrote the paper. All authors discussed the results and commented on the manuscript.

\section{Acknowledgements}

We would like to thank Dr. Zhenying Shi and Dr. Maoyin Li for his critical reading on the manuscript

\section{References}

1. Rodriguez RE, Mecchia MA, Debernardi JM, Schommer C, Weigel D, Palatnik JF: Control of cell proliferation in Arabidopsis thaliana by microRNA miR396. Development 2010, 137(1):103.

2. Sarojam R, Sappl PG, Goldshmidt A, Efroni I, Floyd SK, Eshed Y, Bowman JL: Differentiating Arabidopsis Shoots from Leaves by Combined YABBY Activities. The Plant Cell 2010, 22(7):2113.

3. Zhao W, Li Z, Fan J, Hu C, Yang R, Qi X, Chen H, Zhao F, Wang S: Identification of jasmonic acidassociated microRNAs and characterization of the regulatory roles of the miR319/TCP4 module under root-knot nematode stress in tomato. J Exp Bot 2015, 66(15):4653-4667.

4. Bowman JL, Smyth DR, Meyerowitz EM: Genes directing flower development in Arabidopsis. The Plant Cell 1989, 1(1):37.

5. Kumaran MK, Bowman JL, Sundaresan V: YABBYPolarity Genes Mediate the Repression of KNOX Homeobox Genes in Arabidopsis. The Plant Cell 2002, 14(11):2761.

6. Zhao S-P, Lu D, Yu T-F, Ji Y-J, Zheng W-J, Zhang S-X, Chai S-C, Chen Z-Y, Cui X-Y: Genome-wide analysis of the YABBY family in soybean and functional identification of GmYABBY10 involvement in high salt and drought stresses. Plant Physiology and Biochemistry 2017, 119:132-146.

7. Bowman JL, Smyth DR: CRABS CLAW, a gene that regulates carpel and nectary development in Arabidopsis, encodes a novel protein with zinc finger and helix-loop-helix domains. Development 1999, 126(11):2387.

8. Bowman JL: The YABBY gene family and abaxial cell fate. Current Opinion in Plant Biology 2000, 3(1):17-22.

9. Siegfried KR, Eshed Y, Baum SF, Otsuga D, Drews GN, Bowman JL: Members of the YABBY gene family specify abaxial cell fate in Arabidopsis. Development 1999, 126(18):4117. 
10. Sawa S, Watanabe K, Goto K, Liu YG, Shibata D, Kanaya E, Morita EH, Okada K: FILAMENTOUS FLOWER, a meristem and organ identity gene of Arabidopsis, encodes a protein with a zinc finger and HMG-related domains. Genes Dev 1999, 13(9):1079-1088.

11. Eshed Y, Baum SF, Bowman JL: Distinct Mechanisms Promote Polarity Establishment in Carpels of Arabidopsis. Cell 1999, 99(2):199-209.

12. Villanueva JM, Broadhvest J, Hauser BA, Meister RJ, Schneitz K, Gasser CS: INNER NO OUTER regulates abaxial- adaxial patterning in Arabidopsis ovules. Genes Dev 1999, 13(23):3160-3169.

13. Baker SC, Robinson-Beers K, Villanueva JM, Gaiser JC, Gasser CS: Interactions among genes regulating ovule development in Arabidopsis thaliana. Genetics 1997, 145(4):1109-1124.

14. Klopffleisch K, Phan N, Augustin K, Bayne RS, Booker KS, Botella JR, Carpita NC, Carr T, Chen J-G, Cooke TR et al: Arabidopsis G-protein interactome reveals connections to cell wall carbohydrates and morphogenesis. Mol Syst Biol 2011, 7:532-532.

15. Waites R, Hudson A: phantastica: a gene required for dorsoventrality of leaves in Antirrhinum majus. Development 1995, 121(7):2143.

16. Waites R, Selvadurai HRN, Oliver IR, Hudson A: The PHANTASTICA Gene Encodes a MYB Transcription Factor Involved in Growth and Dorsoventrality of Lateral Organs in Antirrhinum. Cell 1998, 93(5):779-789.

17. Sawa S, Ito T, Shimura Y, Okada K: FILAMENTOUS FLOWER controls the formation and development of arabidopsis inflorescences and floral meristems. The Plant cel/ 1999, 11(1):69-86.

18. Kerstetter RA, Bollman K, Taylor RA, Bomblies K, Poethig RS: KANADI regulates organ polarity in Arabidopsis. Nature 2001, 411(6838):706-709.

19. McConnell JR, Emery J, Eshed Y, Bao N, Bowman J, Barton MK: Role of PHABULOSA and PHAVOLUTA in determining radial patterning in shoots. Nature 2001, 411(6838):709-713.

20. Juarez MT, Twigg RW, Timmermans MCP: Specification of adaxial cell fate during maize leaf development. Development 2004, 131(18):4533.

21. Pekker I, Alvarez JP, Eshed Y: Auxin Response Factors Mediate \&lt;em\&gt;Arabidopsis\&lt;/em\&gt; Organ Asymmetry via Modulation of KANADI Activity. The Plant Cell 2005, 17(11):2899.

22. Eshed Y, Baum SF, Perea JV, Bowman JL: Establishment of polarity in lateral organs of plants. Current Biology 2001, 11(16):1251-1260.

23. Emery JF, Floyd SK, Alvarez J, Eshed Y, Hawker NP, Izhaki A, Baum SF, Bowman JL: Radial Patterning of Arabidopsis Shoots by Class III HD-ZIP and KANADI Genes. Current Biology 2003, 13(20):17681774.

24. Eshed Y, Izhaki A, Baum SF, Floyd SK, Bowman JL: Asymmetric leaf development and blade expansion in Arabidopsis are mediated by KANADI and YABBY activities. Development 2004, 131(12):2997.

25. Tang G, Reinhart BJ, Bartel DP, Zamore PD: A biochemical framework for RNA silencing in plants. Genes Dev 2003, 17(1):49-63. 
26. Yao X, Wang H, Li H, Yuan Z, Li F, Yang L, Huang H: Two types of cis-acting elements control the abaxial epidermis-specific transcription of the MIR165a and MIR166a genes. FEBS Letters 2009, 583(22):3711-3717.

27. Tatematsu K, Toyokura K, Miyashima S, Nakajima K, Okada K: A molecular mechanism that confines the activity pattern of miR165 in Arabidopsis leaf primordia. The Plant Journal 2015, 82(4):596-608.

28. Li H, Xu L, Wang H, Yuan Z, Cao X, Yang Z, Zhang D, Xu Y, Huang H: The Putative RNA-Dependent RNA Polymerase RDR6 Acts Synergistically with ASYMMETRIC LEAVES1 and 2 to Repress BREVIPEDICELLUS and MicroRNA165/166 in Arabidopsis Leaf Development. The Plant Cell 2005, 17(8):2157.

29. Fu Y, Xu L, Xu B, Yang L, Ling Q, Wang H, Huang H: Genetic interactions between leaf polaritycontrolling genes and ASYMMETRIC LEAVES1 and 2 in Arabidopsis leaf patterning. Plant \& cell physiology 2007, 5(48).

30. Hunter C, Willmann MR, Wu G, Yoshikawa M, de la Luz Gutiérrez-Nava M, Poethig SR: Trans-acting siRNA-mediated repression of ETTIN and ARF4 regulates heteroblasty in Arabidopsis. Development 2006, 133(15):2973.

31. Chitwood DH, Nogueira FTS, Howell MD, Montgomery TA, Carrington JC, Timmermans MCP: Pattern formation via small RNA mobility. Genes Dev 2009, 23(5):549-554.

32. Marin E, Jouannet V, Herz A, Lokerse AS, Weijers D, Vaucheret H, Nussaume L, Crespi MD, Maizel A: miR390, Arabidopsis TAS3 tasiRNAs, and their AUXIN RESPONSE FACTOR targets define an autoregulatory network quantitatively regulating lateral root growth. The Plant cel/ 2010, 22(4):11041117.

33. Toriba T, Harada K, Takamura A, Nakamura H, Ichikawa H, Suzaki T, Hirano H-Y: Molecular characterization the YABBY gene family in Oryza sativa and expression analysis of OsYABBY1. Molecular Genetics and Genomics 2007, 277(5):457-468.

34. Lin Z, Li X, Shannon LM, Yeh C-T, Wang ML, Bai G, Peng Z, Li J, Trick HN, Clemente TE et al: Parallel domestication of the Shattering1 genes in cereals. Nature Genetics 2012, 44(6):720-724.

35. Dai M, Hu Y, Zhao Y, Liu H, Zhou D-X: A WUSCHEL-LIKE HOMEOBOX gene represses a YABBY gene expression required for rice leaf development. Plant Physio/2007, 144(1):380-390.

36. Liu H-I, Xu Y-Y, Xu Z-H, Chong K: A rice YABBY gene, OsYABBY4, preferentially expresses in developing vascular tissue. Development Genes and Evolution 2007, 217(9):629-637.

37. Ohmori Y, Toriba T, Nakamura H, Ichikawa H, Hirano H-Y: Temporal and spatial regulation of DROOPING LEAF gene expression that promotes midrib formation in rice. The Plant Journal 2011, 65(1):77-86.

38. Strable J, Wallace JG, Unger-Wallace E, Briggs S, Bradbury PJ, Buckler ES, Vollbrecht E: Maize YABBY Genes drooping leaf1 and drooping leaf2 Regulate Plant Architecture. The Plant cel/ 2017, 29(7):1622-1641.

39. Cong B, Barrero LS, Tanksley SD: Regulatory change in YABBY-like transcription factor led to evolution of extreme fruit size during tomato domestication. Nature Genetics 2008, 40(6):800-804. 
40. Yang Z, Gong Q, Wang L, Jin Y, Xi J, Li Z, Qin W, Yang Z, Lu L, Chen Q et al: Genome-Wide Study of YABBY Genes in Upland Cotton and Their Expression Patterns under Different Stresses. Front Genet 2018, 9:33-33.

41. Gleissberg S, Groot EP, Schmalz M, Eichert M, Kölsch A, Hutter S: Developmental events leading to peltate leaf structure in Tropaeolum majus (Tropaeolaceae) are associated with expression domain changes of a YABBY gene. Development Genes and Evolution 2005, 215(6):313-319.

42. Cao Y, Lang Z, Wang L: Characteristics and Expression Analysis of Transcription Factor YABBY Family in Maize. Journal of Agricultural Science and Technology 2015, 17(01):32-41.

43. Yang C, Li D, Liu X, Ji C, Hao L, Zhao X, Li X, Chen C, Cheng Z, Zhu L: OsMYB103L, an R2R3-MYB transcription factor, influences leaf rolling and mechanical strength in rice (Oryza sativa L.). $B M C$ Plant Biol 2014, 14:158-158.

44. Michelmore R, Marsh E, Seely S, Landry B: Transformation of lettuce (Lactuca sativa) mediated by Agrobacterium tumefaciens. Plant Cell Reports 1987, 6(6):439-442.

45. Clough SJ, Bent AF: Floral dip: a simplified method for Agrobacterium -mediated transformation of Arabidopsis thaliana. The Plant Journal 1998, 16(6):735-743.

\section{Figures}



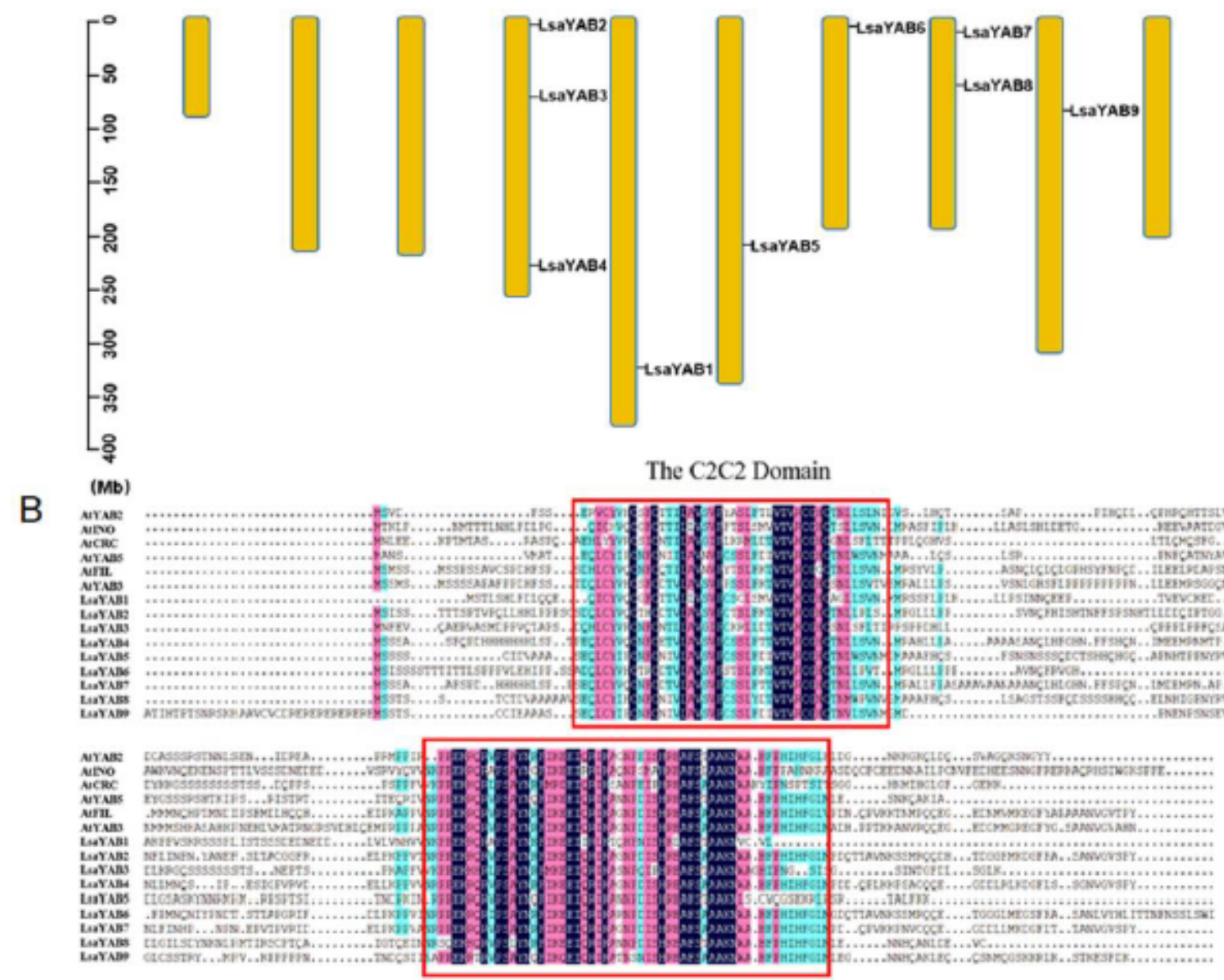

YABBY Domain

\section{Figure 1}

Chromosomal locations and sequence alignments of LsaYABs. (A) Chromosomal locations of LsaYAB genes. Chromosome 0 indicated the sequences that could not be assembled onto any other chromosomes. (B) Alignment of the conserved domains, C2C2 and YABBY, of different YABBY proteins in lettuce and Arabidopsis thaliana. The conserved $\mathrm{C} 2 \mathrm{C} 2$ and $\mathrm{YABBY}$ domains were respectively marked in red rectangles. 


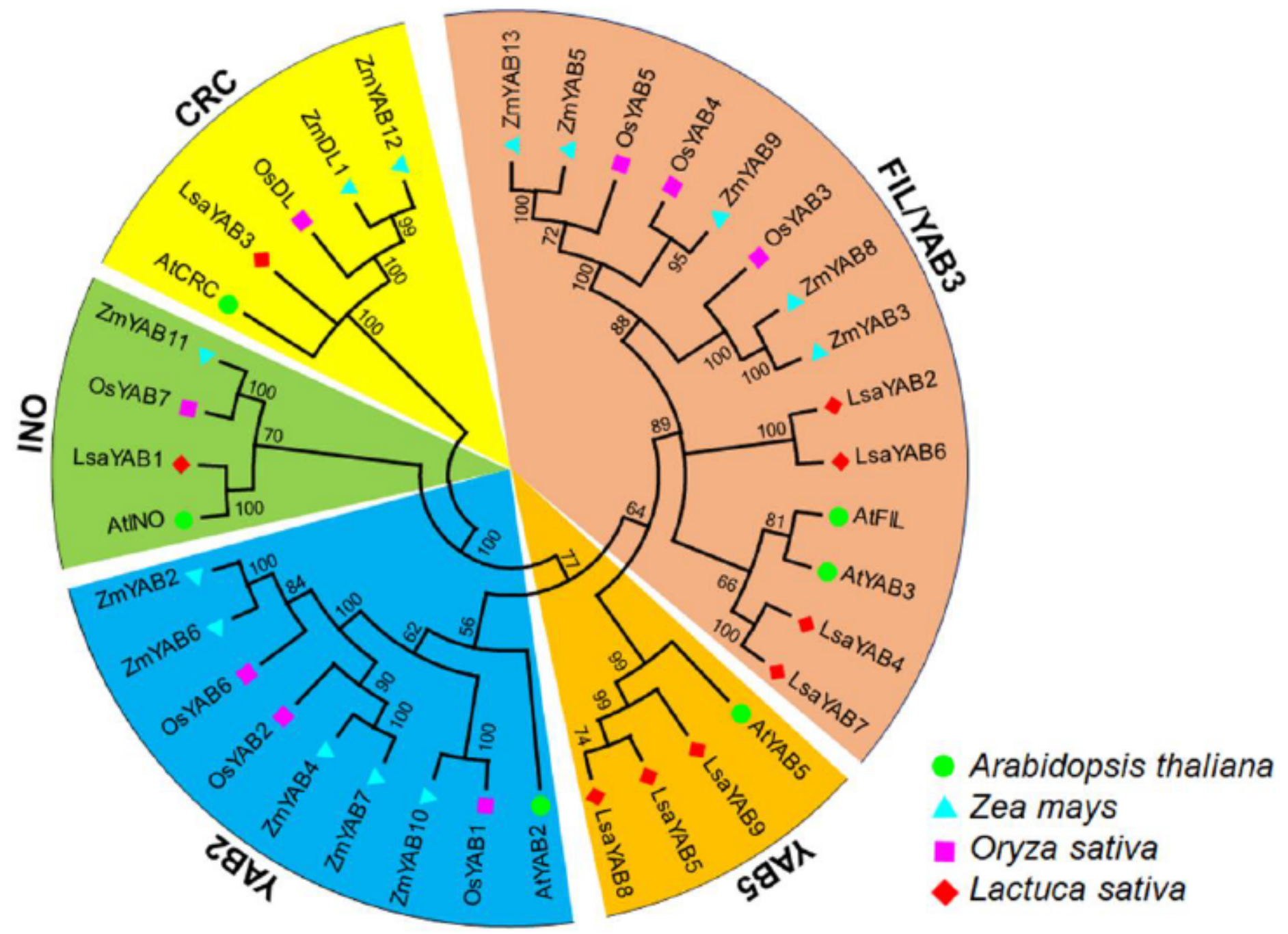

Figure 2

Phylogenetic relationship between YABs from Arabidopsis, rice, maize and lettuce. 

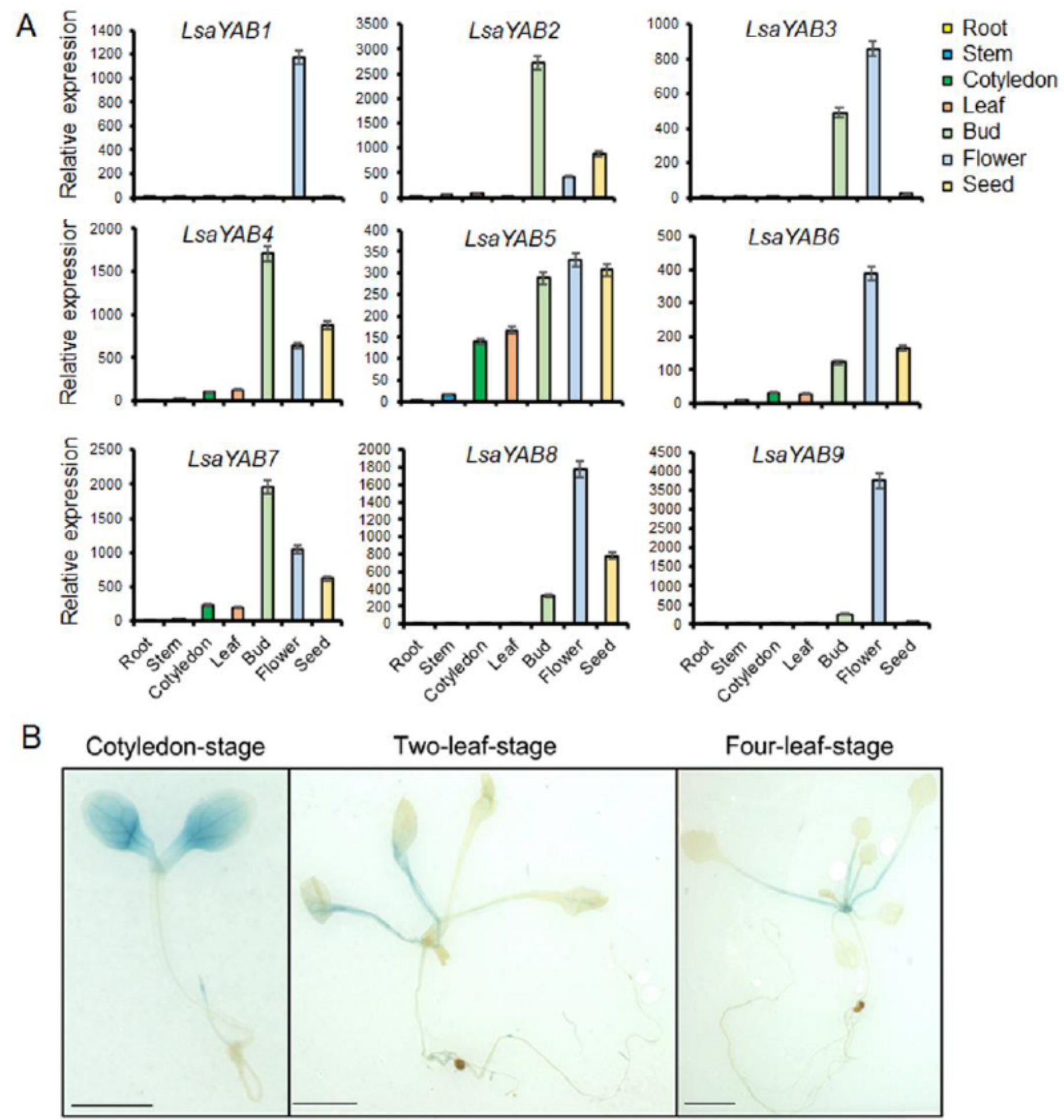

Bar $=500 \mu \mathrm{m}$

\section{Figure 3}

Functional characterization of LsaYAB genes. (A) qRT-PCR analysis of the LsaYAB genes transcript in different tissues of the lettuce 'L3'. Actin was used as a reference gene. (B) GUS staining of pYAB7::GUS transgenic Arabidopsis lines during cotyledon-stage, two-leaf-stage and four-leaf-stage. 
A

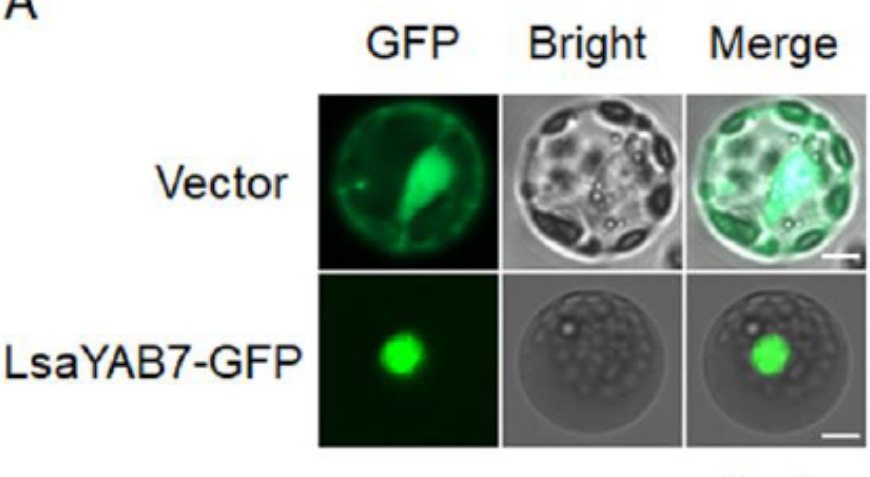

B

$B a r=5 \mu \mathrm{m}$

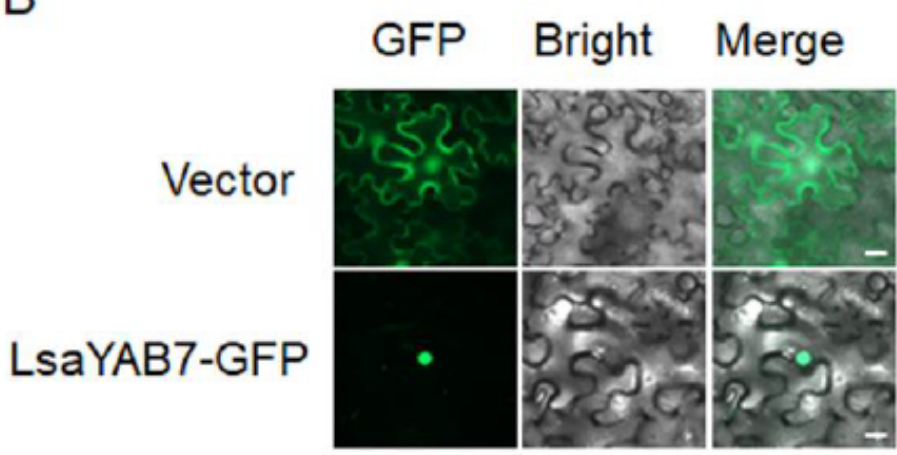

Bar $=20 \mu \mathrm{m}$

C

Positive Control

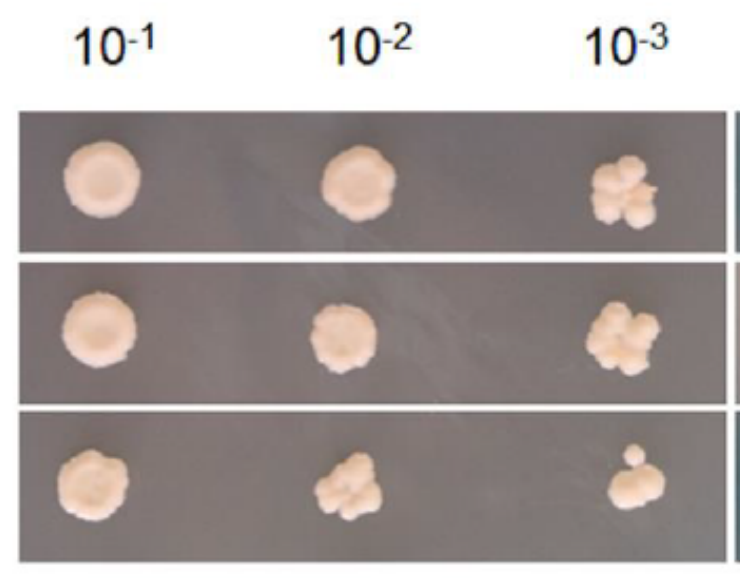

SD/-Trp
$10^{-1}$

$10^{-2}$

$10^{-3}$

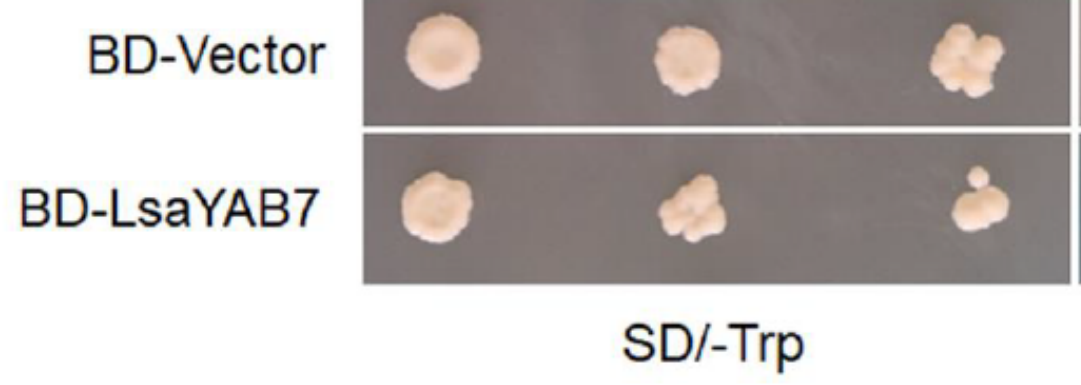

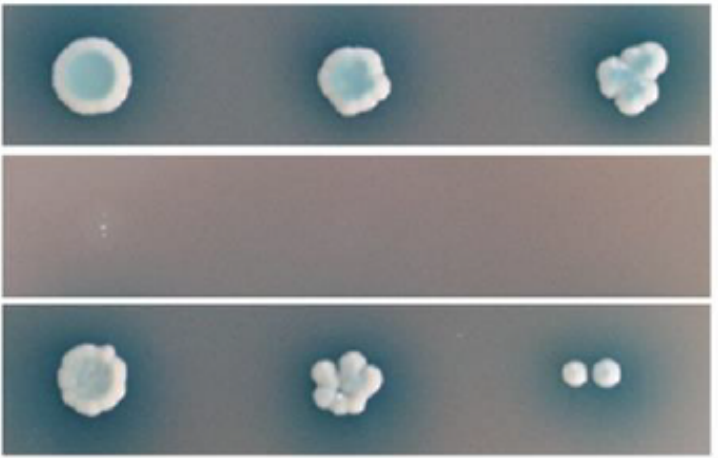

SD/-Trp/ABA/X- $\alpha-G a l$

\section{Figure 4}

Characterization of the LsaYAB7 protein. (A) Subcellular localization of the LsaYAB7-GFP protein in lettuce protoplasts. The fluorescence of LsaYAB7-GFP fusion protein concentrated in the nucleus. Scale Bar $=5 \mu \mathrm{m}$. (B) Subcellular localization of the LsaYAB7-eGFP fusion protein in Nicotiana benthamiana leaves. Scale bar: $20 \mu \mathrm{m}$. (C) Transactivation assay of LsaYAB7 protein in yeast. The full-length of the LsasYAB7 was fused to DNA sequences containing a GAL4 DNA-binding domain in pGBKT7 (BD). The construct was expressed in the yeast strain Y2HGold with PGBDT7-OsMYB103L as a positive control and the void vector as a negative control (pGBKT7). The transformants with different diluted concentrates were dropped on the SD/-trp and SD/-trp/AbA/ X-a-Gal plates. After $2-4$ days at $30^{\circ} \mathrm{C}$, possible transcriptional activation functions of LsaYAB7-full length were observed. 


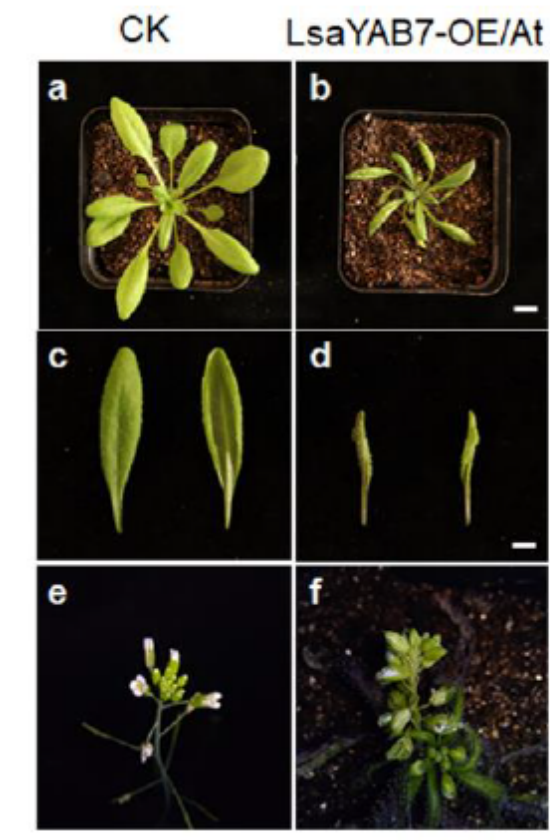

$\mathrm{CK}$ LsaYAB7-OE /At

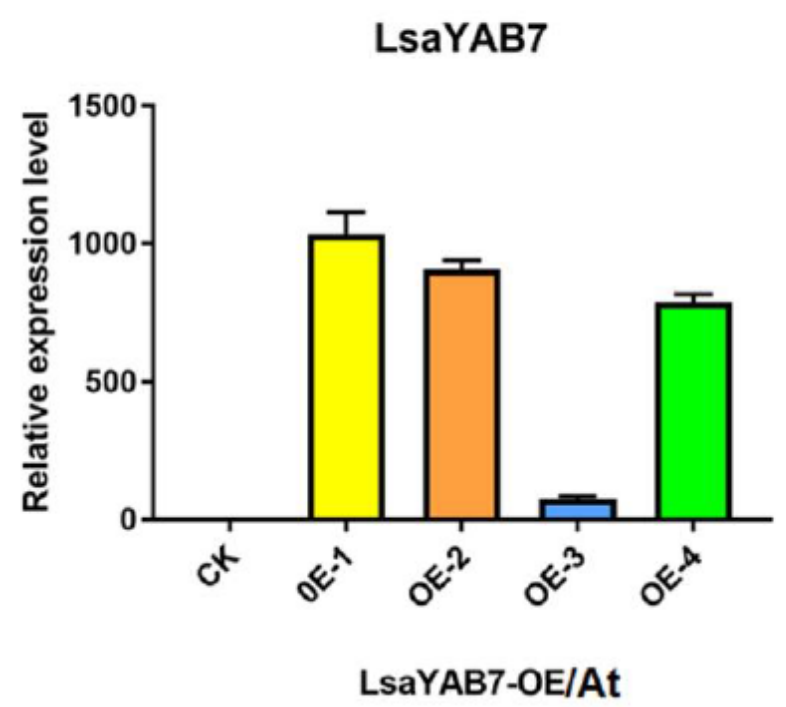

g

\section{Figure 5}

Expression of LsaYAB7 gene in the Arabidopsis LsaYAB7-OE/At plants and phenotyping (A) Expression assay of LsaYAB7 in the LsaYAB7-OE/At lines. The transgenic negative line (CK) was used as negative control. (B) The phenotypes of T1 progeny of LsaYAB7-OE/At lines and transgenic negative line (CK). The rosette stage of CK and LsaYAB7-OE/At line were showed in a and b, respectively. The abaxial and adaxial of leaves in CK and LsaYAB7-OE/At line were exhibited in $c$ and d, respectively. The inflorescence of CK and LsaYAB7-OE/At line were in e and $f$, respectively. The whole seedlings of CK and LsaYAB7$\mathrm{OE} /$ At line at flowering stage were showed in $\mathrm{g}$. 
A

LsaYAB7-OE/lettuce

B

\section{$\begin{array}{lllll}\text { Marker } \mathrm{OE}-1 & \mathrm{OE}-2 & \mathrm{OE}-3 & \mathrm{H}_{2} \mathrm{O} & \text { Vector }\end{array}$}
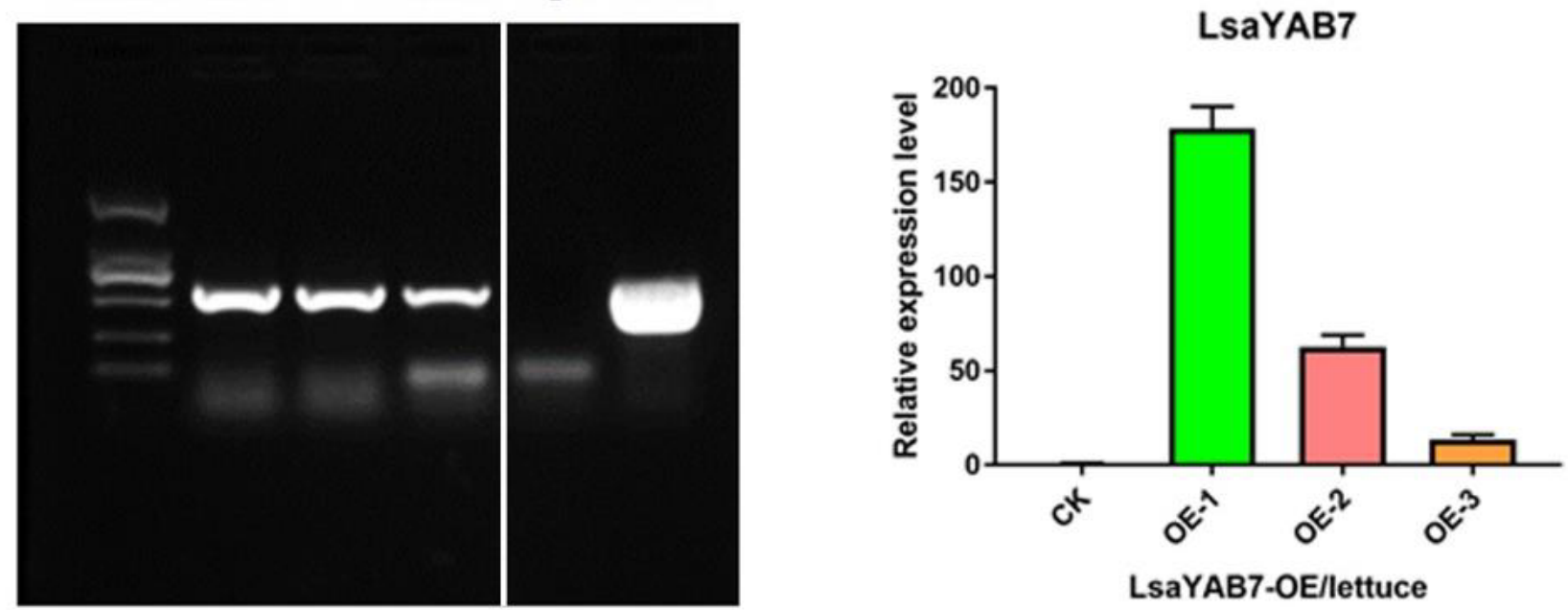

C

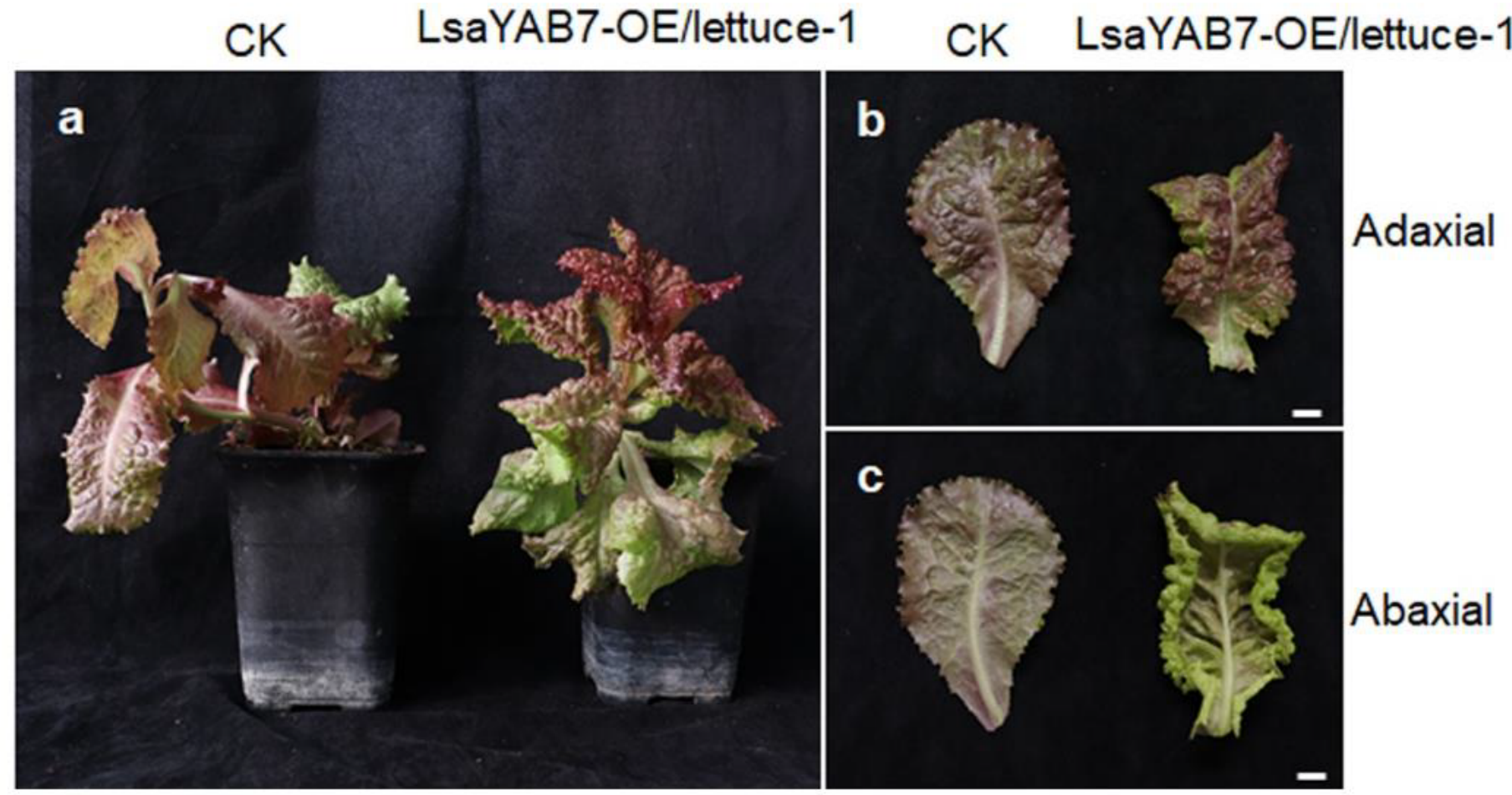

Figure 6

Phenotypes of the over-expression lines of LsaYAB7 in lettuce. (A) Verification of positive overexpression lines of LsaYAB7-OE/lettuce by PCR. The primers for verification were designed from LsaYAB7 and vector, respectively. (B) The relative expression level of LsaYAB7 in LsaYAB7-OE/lettuce lines. The transgenic negative line (CK) was used as negative control. (C) The phenotypes of T1 progeny of LsaYAB7$\mathrm{OE} /$ lettuce and transgenic negative line (CK) in lettuce. The rosette stage of CK and LsaYAB7-OE/lettuce line were showed in a, respectively. The abaxial and adaxial of leaves in CK and LsaYAB7-OE/lettuce line were exhibited in $\mathrm{b}$ and $\mathrm{c}$. 


\section{Supplementary Files}

This is a list of supplementary files associated with this preprint. Click to download.

- additionalfile1.docx

- additionalfile2.xlsx

- additionalfile3.docx 